\title{
Operation of the Silicon Drift Detectors of the ALICE Inner Tracking System during cosmic runs
}

\author{
Francesco Prino* for the ALICE collaboration \\ INFN, Sezione di Torino, Torino, Italy \\ E-mail: prinodto.infn.it
}

Silicon Drift Detectors (SDD) constitute the two intermediate layers of the ALICE Inner Tracking System and provide high-resolution reconstruction of the particle hit on the detector surface as well as energy deposit information for particle identification purposes. The performance of the SDD during the 2008 cosmic run is presented, focusing in particular on the procedures to monitor the relevant calibration parameters (noise, gain, drift speed), on the techniques used for the alignment of the sensors and on the $\mathrm{dE} / \mathrm{dx}$ calibration. First results from the 2009 cosmic run are also presented.

9th International Conference on Large Scale Applications and Radiation Hardness of Semiconductor Detectors, RD09

September 30-October 2, 2009

Florence, Italy

\footnotetext{
* Speaker.
} 


\section{Introduction}

ALICE [1] is a general-purpose detector for the study of $\mathrm{p}-\mathrm{p}, \mathrm{p}-\mathrm{A}$ and heavy-ion collisions at the LHC [2]. The Inner Tracking System (ITS) is the detector of the ALICE central barrel located closest to the beam axis. It allows to improve the momentum and angular resolution for tracks reconstructed in the TPC and to recover particles that are missed by the TPC. Furthermore, it is a key detector for an accurate measurement of the positions of the interaction vertex and of the decay vertices of hyperons and heavy flavoured hadrons. The ITS is composed of six cylindrical layers of silicon detectors, covering the pseudorapidity range $|\eta|<0.9$ and located at radial distances in the range between 3.9 and $43 \mathrm{~cm}$ from the beam axis. The two innermost layers are equipped with pixel detectors (SPD), the two intermediate layers are made of drift detectors (SDD), while double-sided strip detectors (SSD) are used in the two outermost layers. The four layers equipped with SDD and SSD provide also particle identification capability via $\mathrm{dE} / \mathrm{dx}$ measurement. This feature allows to use the ITS also as a standalone spectrometer, able to track and identify particles down to momenta below $200 \mathrm{MeV} / \mathrm{c}$. A data sample of more than 100k atmospheric muons collected in summer 2008 exploiting the SPD FastOR trigger [3] has been used for first track-based alignment of the ITS sensors and charge signal calibration in SDD and SSD. The ITS standalone tracker [2] was adapted to reconstruct the cosmic rays as two back-to-back tracks starting from a pseudo-vertex along the muon trajectory built from the points of the two SPD layers.

In the next sections, the main results from the SDD operation during the cosmic run of 2008 will be presented together with some preliminary performance studies from the ongoing 2009 cosmic run. The drift detectors are described in section 2 together with the readout electronics and the cooling system. The calibration strategy, which is a crucial point for a drift detector, is discussed in section 3. The methods developed for the alignment of the SDD sensors using reconstructed tracks are described in section 4. Finally, charge (dE/dx) calibration is discussed in section 5.

\section{Detector, Cooling and Acquisition}

The ITS layers equipped with drift detectors are composed of linear structures of SDD modules, called ladders, mounted on a carbon fiber support structure to provide full azimuthal coverage. There are 14 ladders with 6 modules each on the inner SDD layer (layer 3 at radius $\approx 15 \mathrm{~cm}$ ), and 22 ladders with 8 modules each on the outer SDD layer (layer 4 , radius $\approx 23.9 \mathrm{~cm}$ ), for a total number of 260 modules. The basic building block of the ladders is the SDD module which consists of one silicon drift sensor, two hybrid circuits hosting the front-end chips and connected via multilayer aluminum-on-kapton microcables to end-ladder LV boards [4]. A microcable specially designed to carry high voltage (up to $2.4 \mathrm{kV}$ ) connects the detector to an end-ladder HV board. The end-ladder LV and HV boards are connected to the power supplies and to the end-ladder data-compression board which collects data from the two hybrids and sends them to the data acquisition system.

The SDD sensors of the ALICE experiment are produced from n-type high-resistivity 300 $\mu \mathrm{m}$-thick Neutron-Transmutation-Doped silicon. A sketch of the layout of a sensor is shown in Fig. 1. The active area of $7.02 \times 7.53 \mathrm{~cm}^{2}$ is split into two drift regions $(\approx 35 \mathrm{~mm}$ long) by a central cathode strip which is biased at a voltage $\mathrm{HV}$ of $\approx 1800 \mathrm{~V}$. In each drift region and on both detector surfaces, $291 \mathrm{p}^{+}$cathode strips with $120 \mu \mathrm{m}$ pitch are implanted. A built-in voltage 


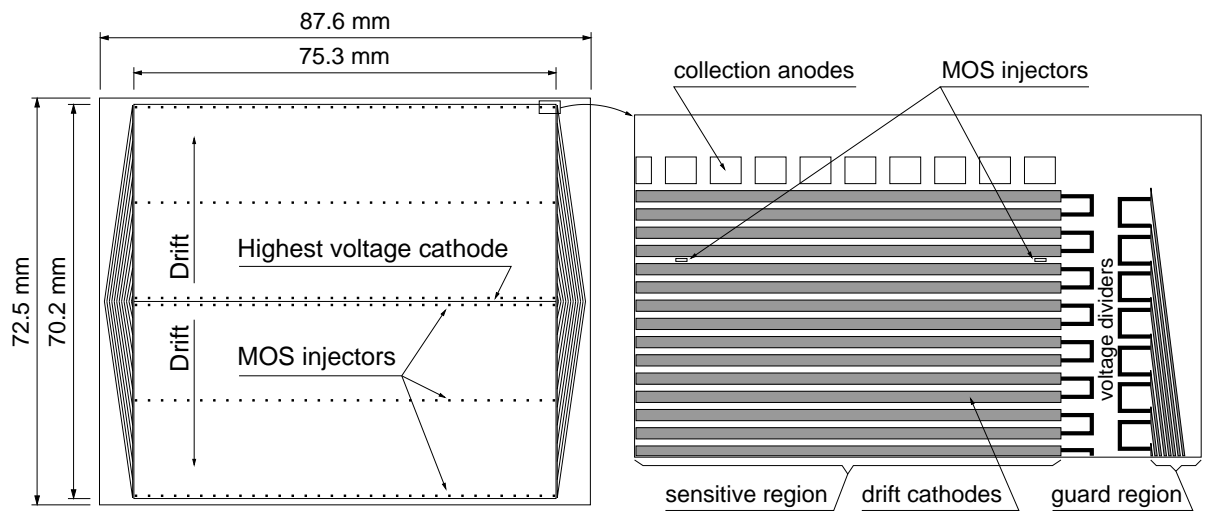

Figure 1: Sketch of an SDD sensor

divider, made of $\mathrm{p}^{+}$implants, biases these cathodes at a gradually decreasing voltage from the $\mathrm{HV}$ applied to the central cathode down to a medium voltage $(\mathrm{MV} \approx-40 \mathrm{~V})$ applied to the last cathodes, thus building a drift field of $\approx 500 \mathrm{~V} / \mathrm{cm}$ parallel to the wafer surface. At the edge of each drift region, the electron cloud is conveyed by means of pull-up cathode strips towards an array of 256 collection anodes ( $294 \mu \mathrm{m}$ pitch) that are connected via micro-cables to the frontend electronics. Hence, in the two drift regions, the electrons, produced by the ionizing particles crossing the sensor, move in opposite directions towards the collection anodes. The coordinate along the ladder (i.e. the $z$ coordinate in the ALICE master reference system) is reconstructed from the centroid of the collected charge along the anodes. The $\mathrm{r} \varphi$ coordinate is reconstructed from the measured drift time. The spatial precision of the SDD detectors, as obtained during beam tests of full-size prototypes, is on average $35 \mu \mathrm{m}$ along the drift direction and $25 \mu \mathrm{m}$ for the anode coordinate. To reach the target resolution along the drift axis, the drift speed (which depends on temperature as $v_{\text {drift }} \propto T^{-2.4}$ ) should be known with $\approx 0.1 \%$ precision. For this purpose, in each of the two drift regions of an SDD module, 3 rows of 33 MOS charge injectors [5] are implanted at known distances from the collection anodes, as sketched in Fig 1. When a dedicated calibration trigger is received, the injector matrix provides a measurement of the drift speed in 33 positions along the anode coordinate for each SDD drift region.

The SDD front-end electronics is based on two ASICs (PASCAL and AMBRA) assembled on a hybrid circuit directly bonded to the sensor and a third ASIC (CARLOS) located at the end of each ladder. For a detailed description, see [6]. The first front-end chip (PASCAL) is a mixedmode ASIC with 64 channels. In each channel the signal coming from one anode is amplified (by a charge sensitive amplifier) and sampled at $40 \mathrm{MHz}$ by an analogue memory with 256 cells. When PASCAL receives a trigger, the memory is frozen, the samples (all of them or one every two depending on the running mode) are digitized by a 10-bit ADC and the data are transmitted to the AMBRA chip, which is a digital four-event buffer performing also baseline equalization and 10-to-8 bit non-linear data compression. Since an AMBRA-PASCAL pair treats the signal of 64 anodes, one hybrid circuit hosts 4 pairs of ASICs to serve the 256 anodes of a drift region. For each sensor, the two front-end hybrids (one per drift region) are connected to the same data-compression board which hosts one CARLOS chip which performs a 2D 2-threshold zero-suppression and sends the data to the DAQ cards in the counting room via optical fibers. In the counting room, $24 \mathrm{VME}$ 

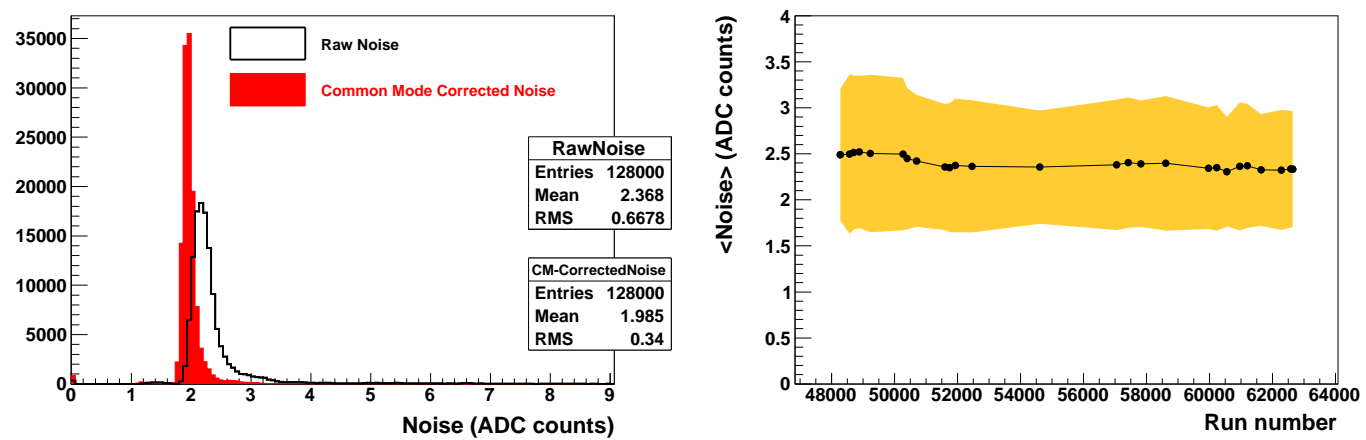

Figure 2: Left: Distribution of noise (raw and corrected for common mode) for all anodes. Right: Average raw noise as a function of run number during 2008 data taking.

boards, named CARLOSrx [7] collect the data coming from the 260 SDD modules and send them to the Local Data Concentrator (LDC) PCs of the ALICE DAQ system. The CARLOSrx allows also to program the front-end ASICs via a JTAG standard interface, to monitor error-flag words in the data flow and (since 2009) to re-format the raw data in a 32-bit per cell fixed-length format aimed at reducing the event size in low-occupancy (cosmic and p-p) runs.

A cooling system with 52 underpressure demineralized-water circuits, each of them with independent flow and pressure control, is coupled to the front-end hybrids and the end-ladder boards, so as to provide a temperature stability of $0.1 \mathrm{~K}$. A dedicated interlock system constantly monitors pressure and flow, thus guaranteeing leak-safety and adequate heat removal.

\section{Calibrating the SDD}

The SDD calibration strategy is based on three types of standalone runs collected periodically (every $\approx 24$ hours) during the data taking and analyzed by dedicated quasi-online algorithms that store the obtained calibration parameters in the Offline Condition Database (OCDB). This information is then retrieved from the OCDB during the offline reconstruction by automatically selecting the most recent set of calibration parameters for the run being analyzed. The first type of calibration run is the pedestal run which allows to measure for each of the $133 \mathrm{k}$ anodes the values of baseline and noise as well as to tag the noisy channels $(\approx 0.5 \%)$. The baselines are then equalized to a common value in the AMBRA chips. An example of distribution of noise (raw and corrected for common mode) for the 250 modules that were in acquisition at the end of 2008 data taking period is shown in the left panel of Fig. 2. The average and r.m.s. values of noise as a function of run number (time) are shown in Fig. 2 right. The $\approx 2.3$ ADC counts of noise match with the design value and should be compared with the 100 ADC counts of charge peak for a cluster produced by a MIP crossing the sensor close to the anodes. The second type of SDD specific calibration run is the pulser run, where a test pulse is sent to the PASCAL input to measure the channel gain and to tag the dead electronic channels $(\approx 1 \%)$. The values of gain are used to equalize the signals of the anodes when calculating the spatial coordinates and the total collected charge of the SDD reconstructed points. The distribution of the gain has an r.m.s. $\approx 4 \%$ of the average value, demonstrating a good uniformity of the electronic channels. Also the gain turned out to be stable during 

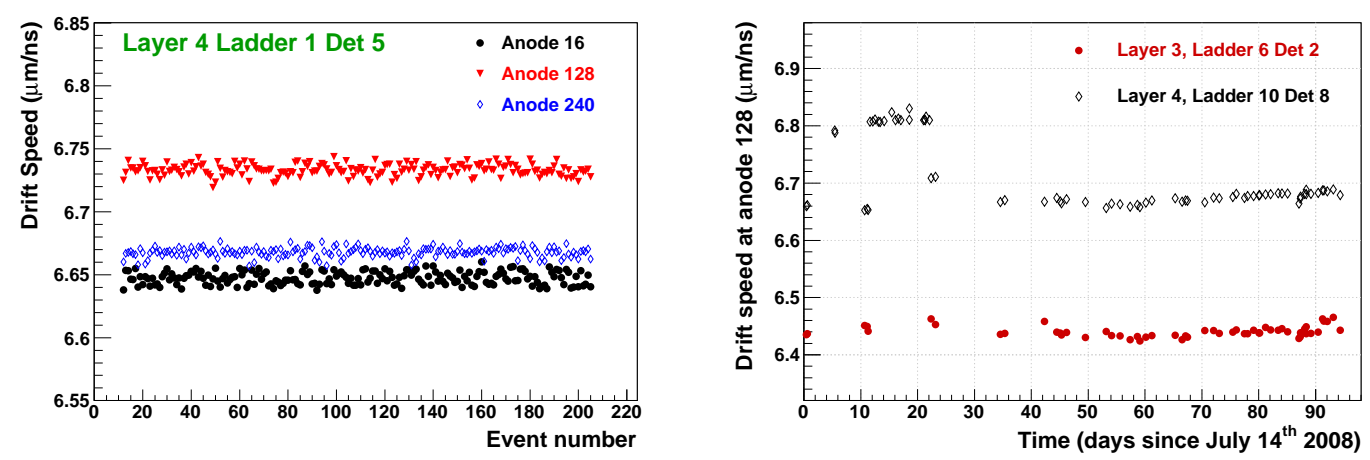

Figure 3: Left: drift speed vs. event number in a special 1 hour run for 3 anodes on one SDD module. Right: drift speed vs. time during 3 months of data taking for one anode on layer 3 and one on layer 4 .

3 months of data taking. The third type of calibration run is the injector run which provides a measurement of the drift speed in 33 positions along the anode coordinate for each SDD drift region by exploiting the MOS charge injectors introduced in section 2. This is a crucial element for the detector calibration because the drift speed depends on temperature (as $T^{-2.4}$ ) and it is therefore sensitive to temperature gradients in the SDD volumes and to temperature variations with time. In the left panel of Fig. 3, the drift speed measured during a special 1 hour run on 3 different anodes of the same SDD sensor is plotted as a function of event number (i.e. time). The different value of speed measured on the 3 anodes is due to a temperature effect: anodes 16 and 240 are located close to the edge of the sensor where the heat sources (the voltage dividers) are located and, as a consequence, the temperature is higher and the electron mobility is lower. The drift speed fluctuations (r.m.s./average value) in 1 hour of running are $\approx 0.07 \%$. In the right panel of Fig. 3 the drift speed is plotted for two anodes (one on layer 3 and one on layer 4) as a function of time for the 3 months of 2008 cosmic run. The lower drift speed on layer 3 calls for a higher temperature in the inner layer. The higher drift speed values $(\approx 6.8 \mu \mathrm{m} / \mathrm{ns})$ observed on layer 4 in the first 22 days of data taking correspond to periods when the layer 3 was off and therefore the temperature on layer 4 was lower. In the period with stable detector configuration (after day 30) a remarkable (within 1\%) stability of the drift speed on a 2-month time scale is observed. Finally, a correction for non-uniformity of the drift field (due to non-linearities in the voltage divider and for few modules also to significant inhomogeneities in dopant concentration) is applied. This correction is extracted from measurements of the systematic deviations between charge injection position and reconstructed coordinates that was performed on all the 260 SDD modules with an infrared laser during the construction phase [8].

\section{Aligning the SDD}

A good knowledge of the real detector geometry is a key point to obtain the design tracking performance of the ITS. Therefore, it is mandatory to align the detector, i.e. to measure the translations and rotations of the real positions of the sensors with respect to the ideal geometry. The ITS alignment procedure starts from the positioning survey measurements performed during the assembling phase and is refined applying track-based alignment methods (such as Millepede [9]) on dedicated samples of tracks from both cosmic ray and $\mathrm{p}-\mathrm{p}$ events. The alignment of SDD detectors 

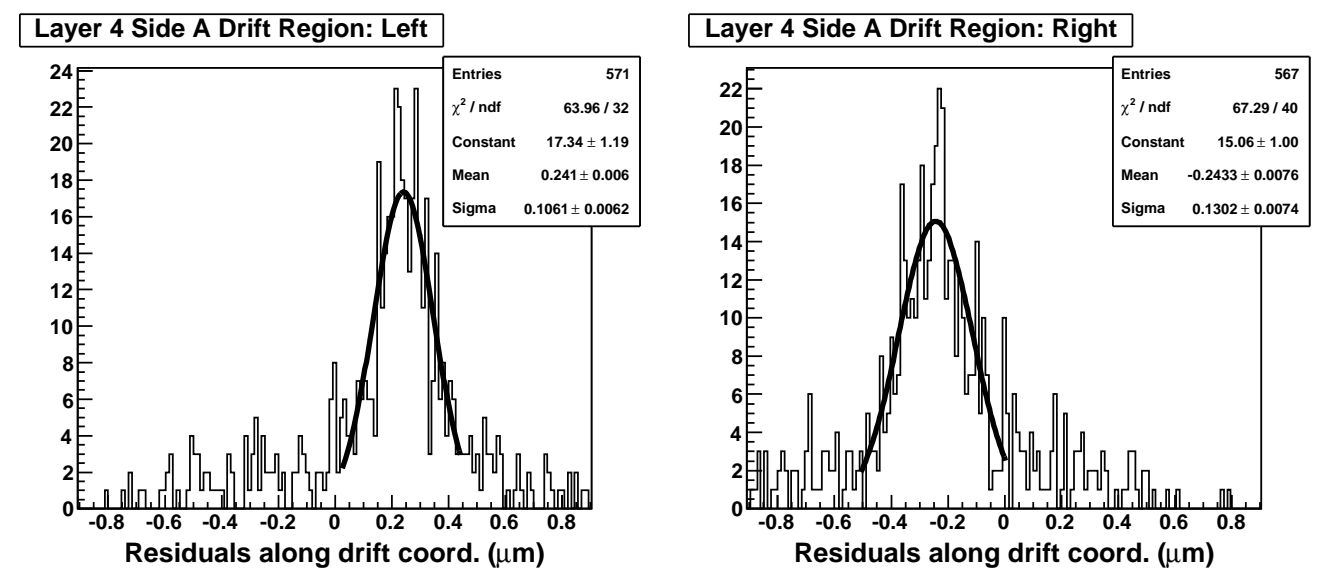

Figure 4: Distribution of track-to-point residuals in the two drift regions of SDD modules of layer 4 side A fitted to Gaussians. The values of the fit parameters are reported in the text box.

for the $r \varphi$ coordinate (reconstructed from the drift time) is complicated by the interplay between the geometrical misalignment and the calibration of drift speed and the time zero $\left(t_{0}\right)$, which is the measured drift time for particles with zero drift distance. The $t_{0}$ parameter accounts for the delays between the time a particle crosses the detector and the time when the front-end chips receive the trigger signal. Two methods have been developed in order to obtain a first estimate of the $t_{0}$ parameter. The first and simpler method consists in extracting the $t_{0}$ from the minimum measured drift time on a large statistics of reconstructed SDD points: the distribution of measured drift times is built and the sharp rising part of the distribution at small drift times is used to extract the $t_{0}$ with an ad-hoc fitting function. The second method measures the $t_{0}$ from the residual distributions along the drift direction between tracks fitted in SPD and SSD layers and the corresponding points reconstructed in the SDD. These distributions, in case of miscalibrated $t_{0}$, show two opposite-signed peaks corresponding to the two drift regions of each SDD module where electrons move in opposite directions (see Fig. 1). The $t_{0}$ can then be calculated from the distance of the two peaks and the drift speed. This second procedure has the advantage of requiring lower statistics because it profits from all the reconstructed points, but it has the drawback of relying on SDD calibration parameters (the drift speed and the correction maps). Moreover, being based on track reconstruction, it might be biased by SPD and SSD misalignments. Depending on the available statistics the determination with these two methods can be done at the level of SDD barrel, SDD ladders or SDD modules. In Fig. 4 the residuals obtained from the first $\approx 2000$ tracks collected in August 2009 is reported. The width of the residual distribution is dominated by the jitter between the time when the muon crosses the detectors and the asynchronous SPD FastOR trigger, which has a readout time of 100 ns. With this initial statistics, it is possible to determine the $t_{0}$ from track-to-point residuals for 4 sub-samples of modules, i.e. separating sensors connected to sides $\mathrm{A}(z>0)$ and $\mathrm{C}(z<0)$ of layers 3 and 4 . For the case of Fig. 4 , a $t_{0}$ of about $422 \pm 9$ ns is obtained. A compatible value is extracted by fitting to an error function the sharp rising-edge of the measured drift time distribution, as it can be seen in Fig. 5. After a first $t_{0}$ calibration with these methods, the Millepede global minimization of track-to-point residuals is run to obtain the re-alignment parameters (rotations and translations) of each SDD sensor. In this calculation, the $t_{0}$ is used as a free global parameter for each of the 260 


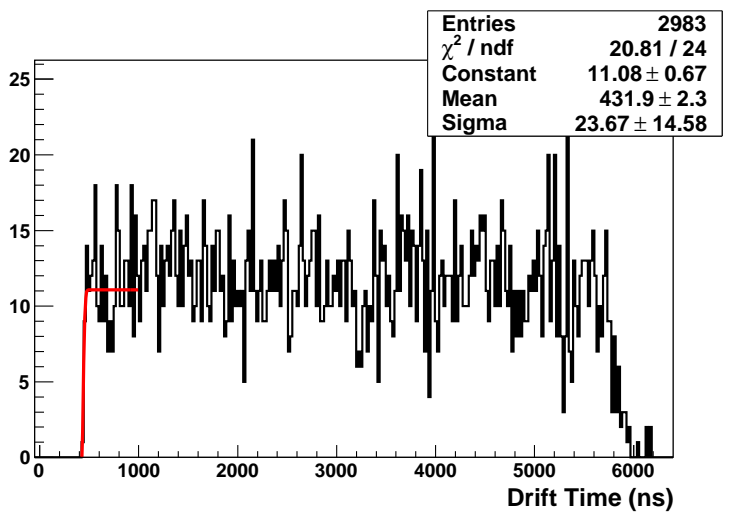

Figure 5: Distribution of measured drift times for SDD modules of layer 4 side A. The sharp rising-edge is fitted to an error function and the resulting values of fit parameters are reported in the text box.

SDD modules. Similarly, the drift speed is considered as a free parameter for those SDD modules with malfunctioning injectors. This resulted to be a powerful method to assess at the same time geometrical realignment and calibration parameters of SDD detector.

\section{5. dE/dx calibration}

The atmospheric muons provide also a sample of ionizing particles for absolute calibration of the $\mathrm{dE} / \mathrm{dx}$ (from ADC units to $\mathrm{keV}$ ). In case of SDD detectors, the collected charge actually depends on drift distance due to the applied zero-suppression: the larger the drift distance, the larger the charge diffusion and consequently the electron cloud develops wider tails which get more easily cut by the zero-suppression algorithm. This effect is shown in the left panel of Fig. 6 where the charge distribution of SDD clusters (i.e. not corrected for track inclination) in two intervals of measured drift time is reported for a sample of atmospheric muon tracks collected without magnetic field during 2008 data taking. The distributions are fitted to a convolution of a Landau and a Gaussian. The conversion factor from ADC to $\mathrm{keV}$ is extracted from detailed simulations of the electronic response of the SDD readout chips and has been cross-checked with measurements performed in the laboratory on few spare SDD modules. In the right panel of Fig. 6, the Most Probable Value (MPV) of energy deposit extracted from Landau+Gaussian fits in 7 bins of drift time is plotted as a function of drift time (closed circles). The MPV decreases with increasing drift time. By applying to the reconstructed cluster charge the correction for the combined effect of diffusion and zero-suppression thresholds, which is extracted from detailed simulations of the detector response, the Landau MPV results to be independent of drift time, as it can be seen from the open triangle points in the right panel of Fig. 6. It should be noted that electron trapping during the drift may also induce a charge dependence on drift time similar to the one observed in Fig. 6. Since these trapping effects are not included in the Monte Carlo simulations used to extract the applied correction, we can conclude that the observed charge dependence on drift time is mainly due to the effect of the thresholds applied by the zero-suppression algorithm. This has also been confirmed by measurements performed in the laboratory on few spare SDD modules, where no dependence of cluster charge on drift time was observed when the zero-suppression was disabled. 

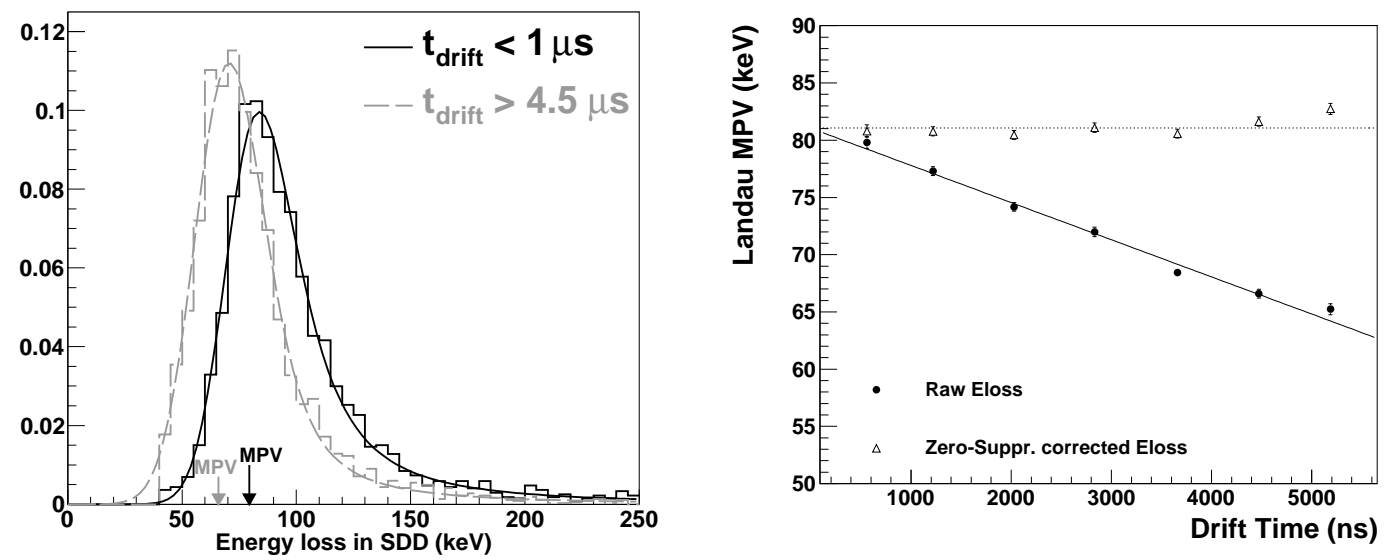

Figure 6: Left: distribution of energy loss for points reconstructed in the SDD detector for two intervals of drift time. Fits with a convolution of a Landau and a Gaussian are superposed. Right: Most Probable Value vs. drift time, with and without correction for zero-suppression effect.

\section{Conclusions}

A successful data taking for commissioning the ALICE Silicon Drift Detector has been carried out in summer 2008 with SPD-triggered atmospheric muons. It allowed to test and validate the calibration strategy, obtaining a good knowledge of the various calibration parameters and assessing in particular the stability of the drift speed during three months of data taking. Moreover, the tools for aligning the SDD sensors and to cope with the interplay between calibration parameters and geometrical misalignments have been developed and tested on cosmic-ray tracks. Finally, the data sample of atmospheric muons allowed to validate the correction for the cluster charge dependence on drift time which is extracted from detailed simulations of the detector response and accounts for the combined effect of charge diffusion and zero-suppression thresholds. As a result, the SDD results to be well performing and ready for $\mathrm{p}-\mathrm{p}$ and $\mathrm{Pb}-\mathrm{Pb}$ collisions.

\section{References}

[1] ALICE collaboration, JINST 3 (2008) S08002.

[2] ALICE collaboration, J. Phys. G: Nucl. Part. Phys. 32 (2006) 1295.

[3] see G. Aglieri Rinella for the ALICE collaboration, in these proceedings.

[4] S. Beolé et al., Nucl. Inst. and Meth. A582 (2007) 733.

[5] A. Rashevsky et al., Nucl. Inst. and Meth. A572 (2007) 125.

[6] A. Rivetti et al., Nucl. Inst. and Meth. A541 (2005) 267.

[7] S. Antinori et al., Nuclear Science Symposium Conference Record, 2006.IEEE Vol. 1, p- 316-320.

[8] G. Batigne et al., JINST 3 (2008) P06004.

[9] V. Blobel, Nucl. Inst. and Meth. A566 (2006) 5. 